\title{
KHẢO SÁT KHẢ NĂNG Ử DỤNG MỘT SỐ PHƯƠNG PHÁP ĐỊNH VỊ GPS TRONG VIỆC THÀNH LẠPP BẢN ĐỒ ĐỊA HİNH ĐÁY BIÊN VEN BỜ Ở VIỆT NAM
}

\author{
ThS. PHAM VĂN QUANG
}

Công ty cổ phần tư vấn, thiết kế và xây dựng 319 - Tổng công ty 319 - Bộ Quốc phòng

\section{Tóm tắt:}

Bài báo trình bày khả năng ứng dụng một số phương pháp định vị GPS trên biển trong việc thành lập Bản đồ địa hình (BĐĐH) đáy biển ven bờ tỷ lệ lớn ở Việt Nam hiện nay như khu vực cửa sông, luồng tàu vào cảng, khu vực cần khảo sát đo vẽ $B \boxminus \boxminus H$ đáy biển ven bờ. Các nghiên cứu về lý thuyết và số liệu đo thực nghiệm đã đánh giá được độ chính xác, ưu điểm, nhược điểm của từng phương pháp định vị từ đó đưa ra kết luận về khả năng ứng dụng của từng phương pháp khi áp dụng định vị trên biển.

\section{1. Đặt vấn đề}

Hiện nay ở nước ta việc thành lập BĐĐH đáy biển ven bờ tỷ lệ lớn để phục vụ thiết kế các công trình biển là rất cần thiết. Định vị mặt bằng của các điểm đo sâu là một trong hai việc chủ yếu để thành lập $\mathrm{BĐĐH}$ đáy biển ven bờ tỷ lệ lớn. Công nghệ GPS đang được ứng dụng rất rộng rãi với nhiều phương pháp đo có thể ứng dụng để xác định vị trí mặt bằng điểm đo như: Phương pháp DGPS, phương pháp đo động tức thời (RTK), đo động tức thời xử lý sau (PPK), phương pháp định vị điểm đơn. Bài báo trình bày kết quả khảo sát độ chính xác của các phương pháp định vị trên bằng thực nghiệm, từ đó đưa ra kết luận về khả năng ứng dụng của mỗi phương pháp trong việc định vị phục vụ công tác thành lập $B \boxminus \boxminus H$ đáy biển ven bờ tỷ lệ lớn ở Việt Nam.

2. Yêu cầu về độ chính xác xác định vị trí mặt bằng điểm đo trong thành lập BĐÐHÐB

\subsection{Quy phạm ngoài nước}

+ Theo quy phạm S-44 của tổ chức thủy đạc quốc tế IHO (International Hydrographic Organization) độ chính xác định vị mặt bằng trên biển được thể hiện trong Bảng 1. (xem bảng 1)

+ Theo Quy phạm của quân đội Hoa Kỳ (USACE - US Army Corps Engineers) độ chính xác định vị mặt bằng trên biển được thể hiện trong Bảng 2. (xem bảng 2)

\subsection{Quy phạm trong nước}

+ Quy định của Bộ Tài nguyên và Môi trường [5]: Sai số trung phương vị trí mặt phẳng của điểm ghi chú độ sâu so với điểm cơ sở (điểm định vị trên bờ) không được vượt quá $\pm 1,0 \mathrm{~mm}$ theo tỷ lệ bản đồ (với tỷ lệ1/10000 là $\pm 10 \mathrm{~m}$ ).

+ Quy phạm đo sâu được sử dụng trong Hải quân nhân dân Việt Nam [1], độ chính xác vị trí điểm đo sâu được tính bằng \pm $0,15 \mathrm{~mm} \cdot \mathrm{M}$ (với M là mẫu số tỷ lệ bản đồ).

3. Sơ lược nguyên lý định vị trên biển bằng công nghệ GPS

Nguyên lý chung của phương pháp định vị trên biển bằng công nghệ GPS là sử dụng 
Bảng 1: Theo quy phạm S-44 của tổ chức thủy đạc quốc tế IHO

\begin{tabular}{|c|c|c|c|c|}
\hline Loại đo đạc & Loại đặc biệt & Loại 1 (d-độ sâu) & Loại 2 (d-độ sâu) & Loại 3 (d-độ sâu) \\
\hline Độ chính xác vị trí điểm & $2 m$ & $5 m+5 \% d$ & $20 m+5 \% d$ & $150 m+5 \% d$ \\
\hline
\end{tabular}

Bảng 2: Theo yêu cầu kỹ thuật của USACE

\begin{tabular}{|c|c|c|c|}
\hline \multirow{3}{*}{ Loại công trình } & \multirow{2}{*}{\multicolumn{2}{|c|}{\begin{tabular}{|c|} 
Bảo đảm an toàn hàng hải và nạo vét luồng lạch \\
loại chất đáy \\
\end{tabular}}} & \multirow{3}{*}{$\begin{array}{l}\text { Các công trình phục vụ } \\
\text { mục đích khác }\end{array}$} \\
\hline & & & \\
\hline & Cứng & Mềm & \\
\hline Độ chính xác vị trí mặt bằng & $<2 \mathrm{~m}$ & $2 m$ & $5 \mathrm{~m}$ \\
\hline
\end{tabular}

Bảng 3: Đặc trưng của một số phương pháp định vị GPS

\begin{tabular}{|l|l|c|c|c|c|}
\hline Phương pháp định vị & Trạm gốc (Base) & Trạm động (Rover) & Toạ độ thu được & Xử lý số liệu & Tầm hoạt động \\
\hline Phương pháp DGPS & Các trạm Beacon & Máy thu Beacon & WGS-84,VN2000 & Tức thời & $300-500 \mathrm{~km}$ \\
\hline Phương pháp RTK & Mốc gốc toạ độ & Hệ thống thu phát tín hiệu & WGS-84,VN2000 & Tức thời & 8-10 km \\
\hline Phương pháp DGPS & Mốc gốc toạ độ & Hệ thống thu tín hiệu & WGS-84 & Xử lý sau & Không giới hạn \\
\hline Phương pháp DGPS & Không cần & Hệ thống thu tín hiệu & WGS-84 & Xử lý sau & Không giới hạn \\
\hline
\end{tabular}

các thiết bị thu tín hiệu vệ tinh kết hợp thuật rong việc thành lập BĐĐH đáy biển ven bờ. toán xử lý nhằm nâng cao độ chính xác định vị. (xem bảng 3)

\subsection{Phương pháp định vị DGPS (Beacon)}

Nếu có 2 máy thu GPS (hoặc nhiều hơn) có thể áp dụng kỹ thuật đo DGPS (Differential GPS), trong đó một máy thu đặt tại điểm đã biết tọa độ gọi là trạm tham chiếu (reference station) còn máy thu khác thì di chuyển (máy động) và sẽ được xác định tọa độ với điều kiện tại cả hai trạm số vệ tinh chung quan sát không ít hơn 4 .

Hiện nay tại Việt Nam đã xây dựng được các trạm phát sóng BEACON MSK tại Đồ Sơn, Cao Bằng, Đà Nẵng, Vũng Tầu, Kiên Giang... Trạm cải chính mặt đất đặt tại Vũng Tàu do hãng FUGRO xử lý và điều hành, hệ thống chăm sóc khách hàng do Công ty IMECOSUM đảm nhận. Với hệ thống các trạm phát sóng BEACON tất cả các vùng biển ven bờ của Việt $\mathrm{Nam}$ đều có thể thu nhận tín hiệu để xác định vị trí mặt bằng

\subsection{Phương pháp GPS động tức thời} (RTK)

Đo GPS động tức thời (RTK: Read Time Kinematic GPS). Phương pháp này cho phép giải được toạ độ điểm đặt máy trạm động ngay tại thực địa nhờ việc xử lý tức thời số liệu thu vệ tinh tại trạm cố định và trạm di động trên bộ xử lý số liệu chuyên dụng đi kèm với trạm động tại thực địa nhưng chỉ cần thu tín hiệu vệ tinh thời gian ngắn ít nhất 1 trị đo (1 Epoch). Nếu khu đo có các điểm toạ độ trong hệ toạ độ địa phương bất kỳ có thể thực hiện việc đo đạc trong hệ toạ độ địa phương thông qua việc đo quy chuyển hệ thống toạ độ (Calibration). Phương pháp này cần phải có hệ thống truyền số liệu (Radio Link) để truyền liên tục số liệu thu được tại trạm tĩnh đến thiết bị xử lý số liệu tại trạm động. Với mỗi cặp thu phát Radio Link phạm vi hoạt động của thiết bị cỡ $10 \mathrm{~km}$. 


\subsection{Phương pháp GPS động xử lý sau (PPK)}

Phương pháp GPS động xử lý sau (PPK - Postprocessing Kinematic GPS). Phương pháp này cho phép thu nhận toạ độ điểm đo có độ chính xác cỡ $\mathrm{cm}$ trên cơ sở xử lý số liệu thu vệ tinh tại trạm cố định và trạm di động trên phần mềm xử lý số liệu chuyên dụng sau khi đo thực địa. Thời gian thu tín hiệu vệ tinh ngắn (tối thiểu trị đo). Phương pháp này không cần đến hệ thống Radio Link truyền số liệu.

\subsection{Phương pháp định vị điểm đơn}

Phương pháp này sử dụng các máy thu GPS để xác định vị trí mặt bằng của điểm, trong quá trình đo có đo vào các điểm song trùng đã biết tọa độ trong hệ tọa độ VN2000 để chuyển kết quả về hệ tọa độ VN2000. Nhờ các điểm song trùng với phần mềm chuyển đổi. Phương pháp này thực hiện được ở mọi nơi khi thu được tín hiệu GPS và chỉ cần 1 máy thu.

\section{Tổ chức đo đạc thực nghiệm}

Để đưa ra kết luận về độ chính xác của các phương pháp định vị, tác giả đã bố trí đo đạc thực nghiệm tại sân vận động Quốc gia Mỹ Đình quận Nam Từ Liêm thành phố Hà Nội.

Tọa độ các điểm GPS1 và GPS2 được dẫn về từ 2 mốc tọa độ Quốc gia, số liệu đo được thực hiện trong 2 ngày 25 và 26 tháng 9 năm 2014. (xem bảng 4)

Tại khu vực đo, ngoài 2 điểm GPS1 và GPS2 còn có 45 điểm đo kiểm tra được bố trí theo sơ đồ hình 1. (xem hình 1)

Tọa độ các điểm đo kiểm tra được xác định bằng 5 phương pháp: Phương pháp toàn đạc, phương pháp định vị vi phân DGPS (Beacon), phương pháp GPS động

Bảng 4: Tọa độ điểm mốc Quốc gia

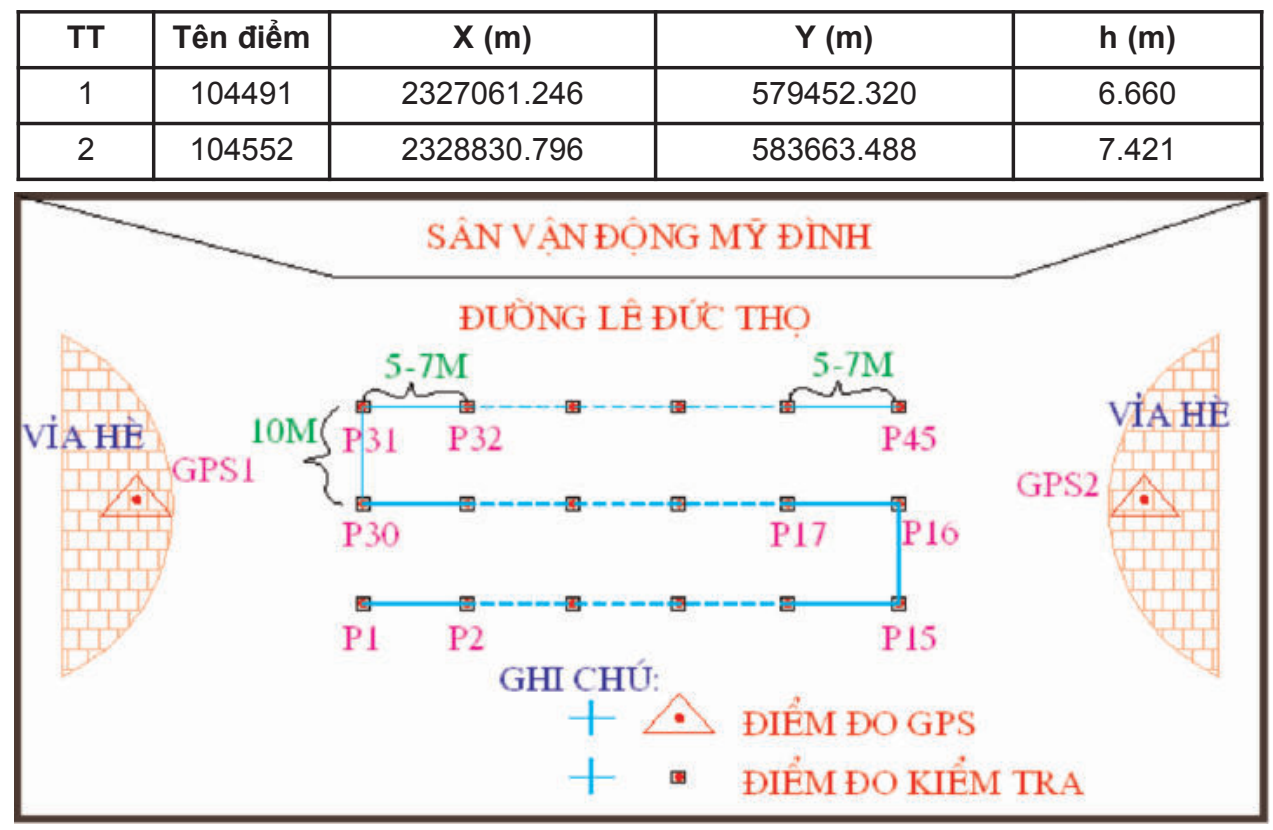

Hình 1: So đồ bố trí đo thực nghiệm 
Bảng 5: Máy móc thiết bị đo thực nghiệm

\begin{tabular}{|l|l|c|l|c|l|}
\hline TT & \multicolumn{1}{|c|}{ Tên thiết bị } & TT & \multicolumn{1}{|c|}{ Tên thiết bị } & TT & \multicolumn{1}{|c|}{ Tên thiết bị } \\
\hline 01 & Máy thu GPS Trimble R7 & 04 & Bộ điều khiển TCS & 07 & Máy TĐĐT DTM-551 \\
\hline 02 & Bộ thu phát Radio link & 05 & Máy tính chuyên dụng & 08 & Máy bộ đàm \\
\hline 03 & Máy thu trạm động, trạm tĩnh & 06 & Bộ nguồn di động & 09 & Các thiết bị khác \\
\hline
\end{tabular}

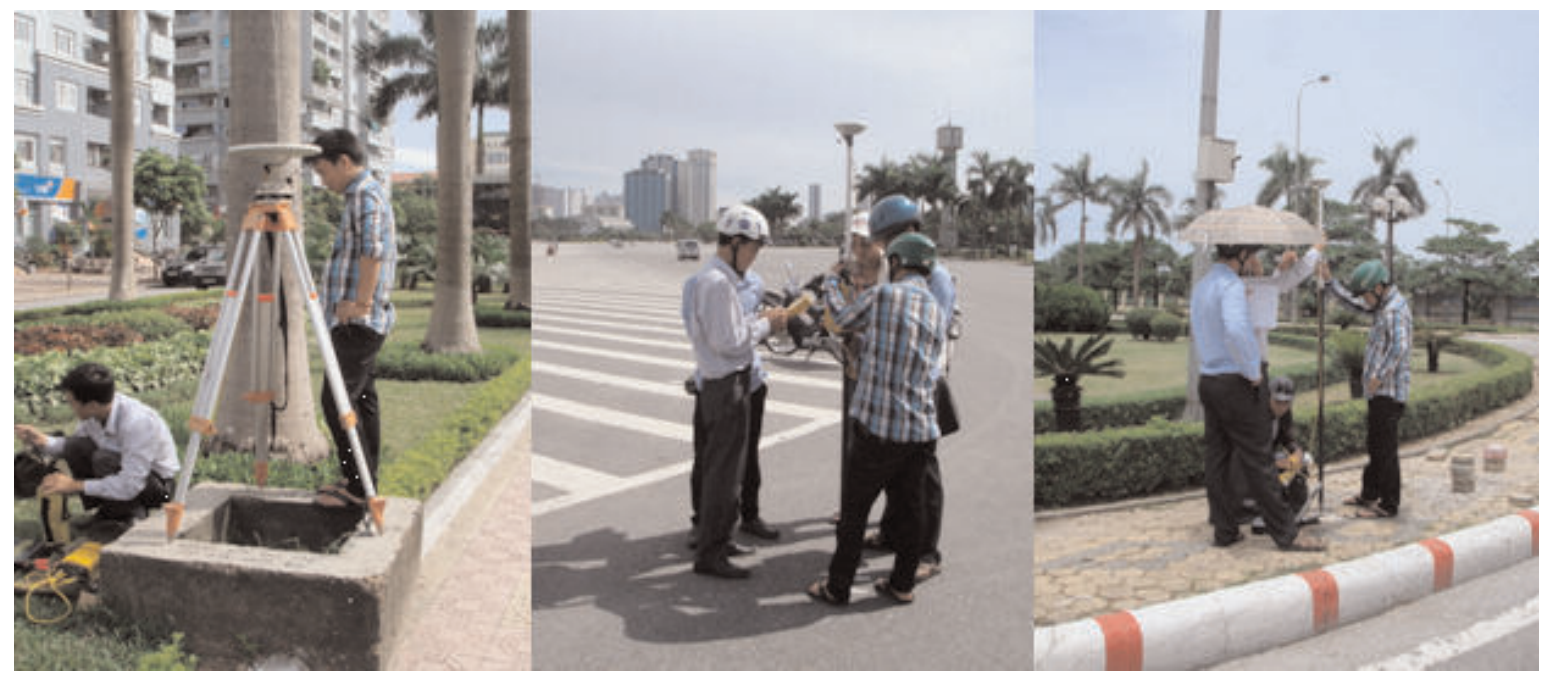

Hình 2: Tổ chức đo thực nghiệm tại sân vận động Quốc gia Mỹ Đình

tức thời (RTK), phương pháp GPS động xử lý sau (PPK) và phương pháp định vị điểm đơn. (xem bảng 5 và xem hình 2)

Tại hiện trường bố trí đo tọa độ các điểm từ $\mathrm{P} 1, \mathrm{P} 2, \ldots$ đến $\mathrm{P} 45$ bằng 5 phương pháp trong đó phương pháp toàn đạc được đo bằng máy TĐĐT DTM-551, kết quả tọa độ các điểm thu được tại hiện trường và xử lý trong nhà được thể hiện trong bảng 6 . (xem bảng 6)

Từ các kết quả ở bảng 6 , coi tọa độ của các điểm đo bằng phương pháp toàn đạc là một trị đo và tọa độ của các điểm cùng tên xác định bằng các phương pháp định vị đo được cũng là một trị đo có cùng độ chính xác. Theo [2] ta tính được sai số trung phương của phép đo áp dụng theo công thức:

$$
m=m_{T D}=m_{P P: G P S}=\frac{1}{2} \sqrt{\frac{[d d]}{n}}
$$

Thay các giá trị độ lệch tính được trong bảng 6 cho từng phương pháp định vị vào (1) ta được:

$$
\begin{aligned}
& \mathrm{m}_{\text {Beacon }}=0.396 \mathrm{~m} ; \\
& \mathrm{m}_{\text {RTK }}=0.041 \mathrm{~m} ; \\
& \mathrm{m}_{\text {PPK }}=0.032 \mathrm{~m} ; \\
& \mathrm{m}_{\text {ĐVĐ }}=0.248 \mathrm{~m}
\end{aligned}
$$

\section{Kết luận}

Với kết quả thu được trong (2) có thể đi đến các kết luận:

- Phương pháp GPS động xử lý sau (PPK) có độ chính xác tốt nhất.

- Phương pháp GPS động tức thời (RTK) và phương pháp GPS động xử lý sau (PPK) có thể dùng trong định vị đo vẽ $\mathrm{B} \boxminus \boxminus H$ đáy biển ven bờ tỷ lệ 1:500. 


\section{Bảng 6: Kết quả tọa độ các điểm kiểm tra}

\begin{tabular}{|c|c|c|c|c|c|c|c|c|c|c|c|c|c|c|}
\hline $\begin{array}{l}\text { Tên } \\
\text { điểm }\end{array}$ & PP TE & כ छт & PP BE & ACON & d-Be & PP R & RTK & d-RTK & PP F & & d-PPK & PP Đा̣NH V!̣ & ĐIÊMM ĐƠN & d-WGS \\
\hline GPS1 & 2325232.767 & 579494.386 & 2325231.900 & 579494.650 & 0.906 & 2325232.694 & 579494.387 & 0.073 & 2325232.705 & 579494.410 & 0.066 & 2325232.767 & 579494.386 & 0.000 \\
\hline GPS2 & 2325582.798 & 579482.497 & 2325582.666 & 579482.373 & 0.181 & 2325582.717 & 579482.511 & 0.082 & 2325582.705 & 579482.497 & 0.093 & 2325582.798 & 579482.497 & 0.000 \\
\hline $\mathrm{P} 1$ & 2325257.607 & 579501.816 & 2325257.629 & 579501.214 & 0.602 & 2325257.537 & 579501.839 & 0.074 & 2325257.561 & 579501.847 & 0.055 & 2325257.581 & 579501.848 & 0.041 \\
\hline P2 & 2325263.521 & 579501.311 & 2325263.694 & 579500.601 & 0.731 & 2325263.457 & 579501.332 & 0.067 & 2325263.489 & 579501.332 & 0.038 & 2325263.508 & 579501.315 & 0.014 \\
\hline $\mathrm{P} 3$ & 2325270.151 & 579501.369 & 2325270.140 & 579501.073 & 0.296 & 2325270.072 & 579501.389 & 0.081 & 2325270.104 & 579501.382 & 0.049 & 2325270.148 & 579501.394 & 0.025 \\
\hline P4 & 2325276.622 & 579501.574 & 2325276.681 & 579501.266 & 0.314 & 2325276.545 & 579501.591 & 0.079 & 2325276.565 & 579501.574 & 0.057 & 2325276.609 & 579501.652 & 0.079 \\
\hline P5 & 2325283.146 & 579501.320 & 2325282.839 & 579500.930 & 0.496 & 2325283.062 & 579501.327 & 0.084 & 2325283.055 & 579501.363 & 0.101 & 2325283.125 & 579501.404 & 0.086 \\
\hline P6 & 2325289.993 & 579501.071 & 2325289.525 & 579500.789 & 0.546 & 2325289.910 & 579501.086 & 0.084 & 2325289.926 & 579501.092 & 0.070 & 2325289.977 & 579501.200 & 0.130 \\
\hline P8 & 2325303.009 & 579501.000 & 2325302.804 & 579500.571 & 0.475 & 2325302.931 & 579501.022 & 0.081 & 2325302.949 & 579501.027 & 0.066 & 2325302.968 & 579501.094 & 0.103 \\
\hline P9 & 2325309.835 & 579500.681 & 2325309.701 & 579500.589 & 0.163 & 2325309.758 & 579500.697 & 0.079 & 2325309.773 & 579500.707 & 0.067 & 2325309.747 & 579500.809 & 0.156 \\
\hline P10 & 2325316.459 & 579500.405 & 2325316.063 & 579500.177 & 0.457 & 2325316.376 & 579500.429 & 0.086 & 2325316.414 & 579500.414 & 0.046 & 2325316.372 & 579501.529 & 1.127 \\
\hline P11 & 2325322.603 & 579500.142 & 2325322.104 & 579499.799 & 0.606 & 2325322.524 & 579500.133 & 0.080 & 2325322.551 & 579500.128 & 0.054 & 2325322.523 & 579500.216 & 0.109 \\
\hline P12 & 2325328.288 & 579499.910 & 2325327.593 & 579499.775 & 0.708 & 2325328.207 & 579499.935 & 0.085 & 2325328.241 & 579499.928 & 0.050 & 2325328.198 & 579500.003 & 0.129 \\
\hline P13 & 2325334.153 & 579499.350 & 2325333.873 & 579499.124 & 0.360 & 2325334.073 & 579499.370 & 0.082 & 2325334.109 & 579499.367 & 0.047 & 2325334.057 & 579499.432 & 0.126 \\
\hline P14 & 2325340.885 & 579499.128 & 2325341.056 & 579498.931 & 0.261 & 2325340.807 & 579499.148 & 0.081 & 2325340.852 & 579499.154 & 0.042 & 2325340.792 & 579499.186 & 0.110 \\
\hline P15 & 2325347.452 & 579498.509 & 2325347.115 & 579498.225 & 0.441 & 2325347.362 & 579498.513 & 0.090 & 2325347.412 & 579498.507 & 0.040 & 2325347.366 & 579498.519 & 0.087 \\
\hline P16 & 2325347.457 & 579488.649 & 2325346.656 & 579488.761 & 0.809 & 2325347.380 & 579488.656 & 0.077 & 325347.409 & 579488.654 & 0.048 & 325347.383 & 579488.674 & 0.078 \\
\hline P17 & 2325340.705 & 579488.912 & 2325340.520 & 579489.021 & 0.215 & 2325340.626 & 579488.917 & 0.079 & 2325340.654 & 579488.913 & 0.051 & 2325340.562 & 579488.882 & 0.146 \\
\hline P18 & 2325334.812 & 579488.917 & 2325334.607 & 579489.912 & 1.016 & 2325334.729 & 579488.940 & 0.086 & 2325334.753 & 579488.934 & 0.061 & 2325334.638 & 579488.832 & 0.194 \\
\hline P19 & 2325328.911 & 579489.369 & 02325328.237 & 579489.850 & 0.828 & 2325328.830 & 579489.384 & 0.082 & 2325328.864 & 579489.385 & 0.050 & 2325328.732 & 579489.232 & 0.225 \\
\hline P20 & 2325322.766 & 579489.853 & 2325322.611 & 579490.839 & 0.998 & 2325322.685 & 579489.867 & 0.082 & 2325322.729 & 579489.843 & 0.038 & 2325322.614 & 579489.648 & 0.255 \\
\hline P21 & 2325316.726 & 579490.367 & 2325316.446 & 579491.239 & 0.916 & 2325316.648 & 579490.387 & 0.081 & 2325316.678 & 579490.384 & 0.051 & 2325309.671 & 579490.182 & 0.233 \\
\hline P22 & 2325309.788 & 579490.838 & 2325309.679 & 579491.742 & 0.911 & 2325309.715 & 579490.858 & 0.076 & 2325309.760 & 579490.853 & 0.032 & 2325302.792 & 579490.621 & 0.247 \\
\hline P23 & 2325302.903 & 579491.192 & 2325302.891 & 579492.177 & 0.985 & 2325302.832 & 579491.219 & 0.076 & 2325302.868 & 579491.207 & 0.038 & 2325302.792 & 579490.949 & 0.267 \\
\hline P24 & 2325296.324 & 579491.592 & 2325296.112 & 579492.380 & 0.816 & 2325296.249 & 579491.607 & 0.076 & 2325296.327 & 579491.587 & 0.006 & 2325296.281 & 0579491.250 & 0.345 \\
\hline P25 & 2325290.255 & 579491.914 & 2325289.648 & 579492.430 & 0.797 & 2325290.187 & 579491.937 & 0.072 & 2325290.201 & 579491.920 & 0.054 & 2325290.507 & 579491.735 & 0.309 \\
\hline P26 & 2325283.041 & 579492.257 & 2325282.790 & 579492.997 & 0.781 & 2325282.974 & 579492.273 & 0.069 & 2325282.982 & 579492.256 & 0.059 & 2325283.073 & 579491.976 & 0.283 \\
\hline P27 & 2325276.104 & 579492.536 & 2325275.803 & 579493.220 & 0.747 & 2325276.035 & 579492.558 & 0.072 & 2325276.067 & 579492.536 & 0.037 & 2325276.082 & 579492.158 & 0.379 \\
\hline P28 & 2325276.104 & 579492.834 & 2325268.680 & 579493.455 & 0.834 & 2325269.161 & 579492.852 & 0.077 & 2325269.173 & 579492.815 & 0.066 & 2325269.143 & 579492.384 & 0.460 \\
\hline P29 & 2325263.100 & 579493.048 & 2325262.719 & 579493.650 & 0.712 & 2325263.024 & 579493.057 & 0.077 & 2325263.040 & 579493.037 & 0.061 & 2325263.015 & 579492.557 & 0.498 \\
\hline P30 & 2325257.094 & 579493.559 & 2325257.006 & 579494.193 & 0.640 & 2325257.028 & 579493.541 & 0.068 & 2325257.045 & 579493.515 & 0.066 & 2325257.002 & 579492.958 & 0.608 \\
\hline P31 & 2325256.657 & 579483.917 & 2325255.541 & 579483.332 & 1.260 & 2325256.574 & 579483.913 & 0.083 & 2325256.600 & 579483.929 & 0.058 & 2325256.549 & 579483.303 & 0.624 \\
\hline P32 & 2325262.522 & 579483.468 & 2325261.716 & 579482.330 & 1.395 & 2325262.445 & 579483.485 & 0.079 & 2325262.468 & 579483.471 & 0.054 & 2325262.397 & 579482.864 & 0.617 \\
\hline P33 & 2325268.734 & 579483.127 & 2325268.692 & 579482.300 & 0.828 & 2325268.658 & 579483.152 & 0.080 & 2325268.657 & 579483.127 & 0.077 & 2325268.611 & 579482.555 & 0.585 \\
\hline P34 & 2325275.562 & 579482.969 & 2325275.432 & 579482.123 & 0.856 & 2325275.487 & 579482.988 & 0.077 & 2325275.505 & 579482.963 & 0.057 & 2325275.452 & 579482.325 & 0.654 \\
\hline P35 & 2325282.577 & 579482.745 & 2325282.349 & 579481.942 & 0.835 & 2325282.502 & 579482.747 & 0.075 & 2325282.504 & 579482.726 & 0.075 & 2325282.427 & 579482.045 & 0.715 \\
\hline P36 & 2325289.955 & 579482.324 & 2325289.723 & 579481.504 & 0.852 & 2325289.884 & 579482.347 & 0.075 & 2325289.910 & 579482.320 & 0.045 & 2325289.804 & 579481.643 & 0.698 \\
\hline P37 & 2325296.488 & 579482.276 & 2325296.606 & 579481.709 & 0.579 & 2325296.420 & 579482.292 & 0.070 & 2325296.422 & 579482.263 & 0.067 & 2325296.373 & 579481.564 & 0.721 \\
\hline P38 & 2325302.897 & 579482.244 & 2325302.905 & 579481.240 & 1.004 & 2325302.816 & 579482.275 & 0.087 & 2325302.817 & 579482.237 & 0.080 & 2325302.758 & 579481.594 & 0.664 \\
\hline P39 & 2325309.544 & 579481.904 & 2325309.449 & 579481.051 & 0.858 & 2325309.470 & 579481.939 & 0.082 & 2325309.485 & 579481.907 & 0.059 & 2325309.296 & 579481.168 & 0.777 \\
\hline P40 & 2325316.422 & 579481.752 & 02325316.537 & 579480.922 & 0.838 & 2325316.346 & 579481.780 & 0.081 & 2325316.333 & 579481.741 & 0.090 & 2325316.208 & 579481.015 & 0.767 \\
\hline P41 & 2325322.829 & 579481.758 & 2325323.184 & 579480.750 & 1.069 & 2325322.763 & 579481.787 & 0.072 & 2325322.713 & 579481.731 & 0.119 & 2325322.584 & 579481.011 & 0.786 \\
\hline P42 & 2325328.884 & 579481.758 & 2325329.356 & 579480.666 & 0.854 & 2325328.815 & 579481.424 & 0.083 & 2325328.810 & 579481.378 & 0.074 & 2325328.638 & 579480.655 & 0.764 \\
\hline P43 & 2325334.995 & 579480.701 & 2325335.273 & 579479.820 & 0.924 & 2325334.903 & 579480.726 & 0.095 & 2325334.932 & 579480.706 & 0.063 & 2325334.754 & 579479.942 & 0.796 \\
\hline P44 & 2325340.727 & 579480.565 & 2325341.339 & 579480.022 & 0.818 & 2325340.635 & 579480.595 & 0.097 & 2325340.658 & 579480.553 & 0.070 & 2325340.478 & 579479.810 & 0.795 \\
\hline P45 & 2325347.395 & 579479.624 & 2325347.873 & 579478.870 & 0.893 & 2325347.312 & 579479.640 & 0.085 & 2325347.317 & 579479.600 & 0.082 & 2325347.162 & 579478.860 & 0.798 \\
\hline
\end{tabular}


- Phương pháp định vị điểm đơn sau đó dùng điểm song trùng quy chuyển về hệ tọa độ VN-2000 có thể dùng trong định vị đo vẽ $\mathrm{B} \boxminus \boxminus H$ đáy biển ven bờ tỷ lệ 1:2000.

- Phương pháp định vị DGPS (Beacon) của Việt Nam chỉ áp dụng trong định vị đo vẽ $B \boxminus \boxminus H$ đáy biển ven bờ tỷ lệ 1:5000; tỷ lệ $1: 10.000 \ldots$

- Tầm hoạt động của mỗi phương pháp cũng liên quan đến độ chính xác, khi trạm thu tín hiệu ở xa các trạm xử lý số liệu gốc hiệu chỉnh phát đi (trạm Beacon, trạm Base) thì độ chính xác cũng giảm theo, với phương pháp GPS động tức thời (RTK) có thể không thu được tín hiệu, do đó phương pháp này chỉ áp dụng khi đo đạc thành lập $\mathrm{BĐĐH} \mathrm{đáy} \mathrm{biển} \mathrm{ven} \mathrm{bờ.}$

\section{Tài liệu tham khảo}

[1]. Đặng Nam Chinh (2010), Nghiên cứu hoàn thiện các chỉ tiêu kỹ thuật và quy trình công nghệ đo đạc biển ở Việt Nam, Báo cáo tổng kết đề tài $\mathrm{KH \& CN}$ cấp bộ (Bộ Giáo dục và Đào tạo). Mã số: B-2007-02-35, 8/2010.

[2]. Hoàng Ngọc Hà, Trương Quang
Hiếu, 1999. Cơ sở toán học xử lý số liệu trắc địa. NXB GTVT, Hà Nội.

[3]. Phan Văn Hiến và nnk, 1999. Trắc địa công trình, NXB GTVT, Hà Nội.

[4]. Quyết định số: 180/1998/QĐ-ĐC ngày 31 tháng 03 năm 1998 của Tổng cục Trưởng tổng cục Địa chính.

[5]. U.S Army Corps Engineers (2004), Engineering and Design Hydrographic surveying, Department of the Army, Washington DC.

[6]. A Division of C\&C Technologies (2003), C-Nav GPS System Operations Manual, Washington DC.

[7]. Trimble R7 GNSS, USA. O

\section{Trang Web:}

1. www.Trimble.com

2. www.fugro.com.vn

3. www.Eye4software.com

4. www.thsoa.org/hy99

5. www.cwbdiving.com. $\mathrm{O}$

\section{Summary}

\section{Usability survey some GPS positioning method for mapping the coastal seabed topography in Viet Nam}

MSc. Pham Van Quang,

Joint-stock Company consulting, design and construction of 319.

This paper presents the possibility to adopt some method in marine GPS navigation on the establishment of topographic maps of coastal seafloor large percentage in Vietnam today as the estuary, the port shipping lane in the area to survey topographic mapping coastal seabed. The study of the theory and experimental measurement data to assess the accuracy, advantages and disadvantages of each method of positioning from which to draw conclusions about the applicability of each method when applied to locate on the sea. $O$ 